\title{
Densities, Viscosities, and Thermal Conductivities of the Ionic Liquid 7-Methyl-1,5,7-triazabicyclo[4.4.0] dec-5-enium Acetate and Its Mixtures with Water
}

\author{
Zachariah Steven Baird ${ }^{1} \cdot$ Petri Uusi-Kyyny ${ }^{1} \cdot$ Artur Dahlberg $^{1}$. \\ Daniel Cederkrantz ${ }^{2} \cdot$ Ville Alopaeus $^{1}$
}

Received: 4 August 2020 / Accepted: 18 September 2020 / Published online: 1 October 2020

(c) The Author(s) 2020

\begin{abstract}
7-Methyl-1,5,7-triazabicyclo[4.4.0]dec-5-enium acetate (mTBD acetate) is a protic ionic liquid that is being investigated for use in industrial processes, such as for producing textiles from cellulose. To aid in designing such processes, we have measured the densities, viscosities, and thermal conductivities of mTBD acetate and aqueous mixtures containing mTBD acetate. We also investigated how excess amounts of mTBD or acetic acid affect the density, and found that in general an excess of either component decreases the density. However, when no water is present, the sample with excess acetic acid actually has a slightly higher density than when there is an equimolar amount of acid and base. The maximum density occurs when some water is present (around 30-40 mol\%). We also modeled the density data using the ePCSAFT equation of state and provide simple correlations for calculating the viscosity and thermal conductivity of these mixtures.
\end{abstract}

Keywords Density · ePC-SAFT · Ionic liquid · 7-Methyl-1,5,7-triazabicyclo[4.4.0] dec-5-enium acetate $\cdot \mathrm{mTBD} \cdot$ Thermal conductivity $\cdot$ Viscosity

\section{Introduction}

The ionic liquid 7-methyl-1,5,7-triazabicyclo[4.4.0]dec-5-enium acetate (mTBD acetate) has the ability to dissolve cellulose, and this can be used to produce more valuable products [1]. One example is the production of textile fibers from biomass in the IONCELL-F process $[2,3]$. To design and operate such processes, information

Petri Uusi-Kyyny

petri.uusi-kyyny@aalto.fi

1 Department of Chemical and Metallurgical Engineering, Aalto University, P.O. Box 16100, 00076 Espoo, Finland

2 Thermtest Europe AB, 43268 Veddige, Sweden 
on the thermophysical properties of mTBD acetate, and its mixtures with water, are needed.

ILs are loosely defined as ionic compounds that have a melting point lower than $100{ }^{\circ} \mathrm{C}$ [4]. Usually one or both of the ions is an organic compound. ILs have received growing attention over the past two decades due to their promising properties. For instance, some have the potential to be more sustainable replacements for the volatile organic compounds currently used as solvents in many processes. Also, there are billions of potential ILs that could be created, and this opens up the possibility of creating solvents with properties tailored for a specific use [4].

Many classes of organic compounds have been studied for more than a century. By contrast, there is much less data available about the thermophysical properties of ILs. For example, the 2018 version of the Dortmund Data Bank contains 8.26 million data points, of which only 0.54 million are for ionic liquids [5, 6]. And, much of the available data are for aprotic ILs, with protic ILs making up a smaller portion [7]. Protic ILs are made by mixing an acid and base together, and a proton transfer reaction between the components leads to the formation of ions. Protic ILs have received more interest lately because they are often cheaper and easier to produce [8].

Additionally, ILs generally have more complicated molecular structures than the molecular solvents that have been the main focus of attention in the past. For this reason it is more difficult to understand and make predictions about the properties of ILs, and additional data on their properties are valuable and needed. This includes data on the properties of ILs in aqueous or organic solutions.

In this article, we present new experimental data on the densities, viscosities, and thermal conductivities of mTBD acetate and its mixtures with water. When measuring the densities, we also measured samples with an excess of mTBD or acetic acid to investigate how these changes in composition affected the density. Due to the equilibrium acid-base reaction in protic ILs, an excess of acid or base can occur in real processes, and it is important to understand how such shifts in the composition affect the properties [9-11]. Such a shift in composition can occur, for example, during evaporation or distillation processes. To our knowledge, no other articles have investigated how changes in the acid-to-base ratio affect the density of the overall solution. This article is a continuation of our earlier research, which presented data on the vapor-liquid equilibrium of the same system [12].

\section{Methods}

\subsection{Sample Preparation}

Information about the chemicals used in this study is summarized in Table 1. Water was purified on site with a Millipore Elix 20 purification system. The first sample of 7-methyl-1,5,7-triazabicyclo[4.4.0]dec-5-ene (mTBD) was synthesized and purified at the University of Helsinki. A second sample was purchased from BOC Sciences, and later this was further purified via distillation (see the description in our earlier article [13]). Two samples of acetic acid were also used. The first sample of acetic 


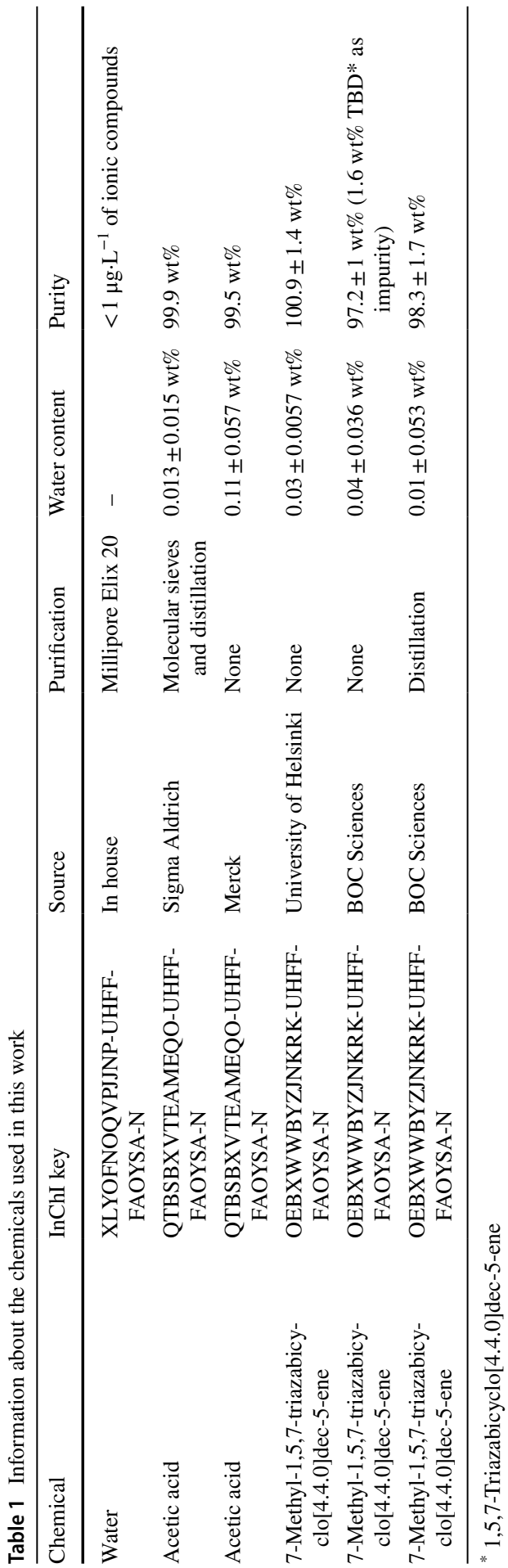


acid was obtained from Sigma Aldrich. It was dried using $3 \AA$ molecular sieves. The acetic acid had a brownish tint after drying, so it was then distilled to get pure acetic acid. The second sample was purchased from Merck and was used without further purification.

The water from the Millipore Elix 20 had a resistance of $18.2 \mathrm{M} \Omega-\mathrm{cm}$ at $25^{\circ} \mathrm{C}$, which corresponds to a concentration of ionic compounds that is less than $1 \mu \mathrm{g} \cdot \mathrm{L}^{-1}$. Karl Fischer titration was used to measure the water contents of the mTBD and acetic acid. The acetic acid was run through a gas chromatograph to assess its purity. The gas chromatograph had a flame ionization detector, and because water does not give a signal with this detector the water content from Karl Fischer titration was taken into account when calculating the purity. The purity of the mTBD was determined using a capillary electrophoresis method described in our earlier article [13].

Initially, the purity of the mTBD from BOC Sciences was thought to be higher, so it was used without purification. After further analyzing the data from the purity analysis, it was found that the actual purity was only $97.2 \mathrm{wt} \%$, and that the mTBD also contained $1.6 \mathrm{wt} \%$ of 1,5,7-triazabicyclo[4.4.0]dec-5-ene (TBD) as an impurity. To see how much the impurities affected the density and viscosity, measurements for the mTBD from BOC Sciences were compared to earlier measurements we had performed with mTBD synthesized at the University of Helsinki [13]. No difference could be detected in the viscosity data, and so the influence of these impurities could be neglected for the viscosity measurements. However, the density measured for the mTBD from BOC Sciences was $1.3 \mathrm{~kg} \cdot \mathrm{m}^{-3}$ higher than the value measured earlier. Therefore, the viscosity data measured with the unpurified sample from BOC Sciences were not remeasured, but the density data were remeasured after purifying the mTBD.

The mTBD was purified via distillation, as described in our earlier article [13]. This was done using a $0.55 \mathrm{~m}$ Vigreux column and a reflux ratio of 3:8. The distilled mTBD had a purity of $98.3 \pm 1.7 \mathrm{wt} \%$. Trace amounts of TBD were still detected in the distilled sample (as well as in the first sample from the University of Helsinki), but the amount was below the limit of quantitation, which was $0.0025 \mathrm{mg} \cdot \mathrm{mL}^{-1}$. The density of the distilled $\mathrm{mTBD}$ was $1063.6 \mathrm{~kg} \cdot \mathrm{m}^{-3}$ at $298.15 \mathrm{~K}$ and $1067.2 \mathrm{~kg} \cdot \mathrm{m}^{-3}$ at $293.15 \mathrm{~K}$, which matches the values from our earlier article at the same level as the uncertainty of the measurements (expanded uncertainty for these measurements is $0.19 \mathrm{~kg} \cdot \mathrm{m}^{-3}$ ) [13]. The condensation temperatures and distillation pressures were recorded during the distillation, and this vapor pressure data were also published in the earlier article [13].

Mixtures of acetic acid, mTBD and water were prepared by mixing the components in a flask. Because acetic acid and mTBD are hygroscopic, they were stored in sealed vials with a septum in the cap. Then the amount needed for a particular mixture was taken using a syringe with a needle. This helped to keep these compounds from coming into contact with moisture in the air. MTBD was added first, and when adding the acetic acid the flask was cooled in an ice bath. This was done because the reaction between $\mathrm{mTBD}$ and acetic acid is exothermic.

When making the mixtures, the amount of each compound added was weighed, and these masses were then used to calculate the composition. A Precisa 410AM-FR balance was used, and the repeatability of the balance was measured to be $0.00085 \mathrm{~g}$. 
Uncertainties for the compositions were calculated based on the repeatability of the balance, and are given in the data file which has been placed in a scientific repository (OSF: https://osf.io/krx5z/). The standard uncertainty of the composition was usually less than $0.1 \mathrm{~mol} \%$.

\subsection{Density Measurements}

Densities were measured using a DMA HP density meter (Anton Paar GmbH, Graz, Austria). The DMA HP density meter was calibrated using air and water. When measuring air the atmospheric pressure, temperature and relative humidity of the lab air were also recorded. Reference values for these compounds were taken from reference equations of state $[14,15]$. For performing calculations with these equations of state CoolProp's [16] Python package was used. The parameters for the calibration equation were optimized using the differential evolution solver [17] implemented in the SciPy package [18] for Python. The root mean squared error was used as the objective function. From the calibration results the standard uncertainty of the device was estimated to be $0.083 \mathrm{~kg} \cdot \mathrm{m}^{-3}$ (expanded uncertainty of $0.19 \mathrm{~kg} \cdot \mathrm{m}^{-3}$ at the $95 \%$ level). Impurities in the samples also affect the density, and for the experimental data this added uncertainty was taken into account. The expanded uncertainty for each point, when accounting for impurities, can be found from the data file on OSF (https://osf.io/krx5z/).

Small bubbles often formed when preparing the samples. We suspect that these bubbles are formed due to a decrease in the gas solubility in water as the ions are added (sometimes called the salting out effect) $[19,20]$. Many of these samples are viscous, which can make it more difficult to remove the bubbles. An important part of sample preparation was removing these bubbles, and this was done by degassing the sample after mixing. To degas, the flask with the sample was connected to a vacuum pump for about 5 to $10 \mathrm{~min}$. The mass was recorded before and after degassing, and this loss was subtracted from the water content of the sample. This was done because vapor-liquid equilibrium measurements showed that the vapor phase consists almost entirely of water [12].

To avoid absorption of water from the air, especially for samples with low water contents, the inlet and outlet of the density meter were closed during the measurements.

mTBD can also hydrolyze [2, 21], and some hydrolysis was observed during the density measurements. For some of the samples, repeat measurements at the same temperature gave different densities, and when measured over time the measured density changed continuously. This was observed only with samples that had no acetic acid or more mTBD than acetic acid. The points where hydrolysis likely occurred have been marked in the data, and generally it occurred at temperatures above $323 \mathrm{~K}$ for these samples. These points were not used in modeling with ePC-SAFT.

\subsection{Viscosity Measurements}

A Brookfield LVDVE230 rotational viscometer was used to measure the viscosities. The temperature was controlled using a water bath. To measure the temperature two RTD temperature probes were placed in the flow of the heating water at the inlet 
and outlet of the measurement cylinder. The average of these two temperatures was taken to be the temperature of the cell. When making a temperature change at least 30 min was allowed to pass before recording values to ensure that the temperature had stabilized. Calibration was performed using a Brookfield viscosity standard (lot number 012610), water and n-hexadecane. Based on the results from calibration, the standard uncertainty was calculated to be $5.4 \%$, and the expanded uncertainty (95\% level) was $12 \%$.

The rotational viscometer we used is open to the atmosphere, and it was not possible to seal it off because there must be at least a little open space at the top to allow the spindle to rotate freely. Therefore, over the course of the experiments some amount of water from the mixture evaporated, which affects the uncertainty of the concentration. We did not visually observe any decrease in the level of the mixture nor did we notice any drift in the viscosity value. We also performed a test with pure water at $363 \mathrm{~K}$ (the highest measurement temperature) to estimate how much might have evaporated. Based on this test and calculations to account for the different vapor pressure at different compositions, we estimate that the expanded uncertainty of the composition is likely $0.005 \mathrm{~mol}$ fraction at the highest temperature $(363 \mathrm{~K})$, and decreases proportionally to the vapor pressure at lower temperatures down to about 0.0003 at $300 \mathrm{~K}$.

\subsection{Thermal Conductivity Measurements}

The method used for measuring the thermal conductivities was described in our earlier article [13]. In summary, a THW-L2 Liquid Thermal Conductivity Meter from Thermtest Inc. was used, which uses the transient hot wire method. In this method, the sensor wire is briefly heated and the change in temperature is measured. The thermal conductivity is then calculated using the Fourier heat conduction equation [22]. The device was calibrated with water, and the expanded uncertainty of the device at the $95 \%$ confidence level was estimated to be $5 \%$.

Some of the samples changed color during the measurements, which could indicate that some reactions occurred. We were not able to reanalyze the samples to check their purities after the measurements, but past measurements have shown that even small amounts of impurities can lead to a color change [13]. We have, however, analyzed samples with similar colors and the concentrations were small enough that the impurities could not be detected using our capillary electrophoresis method. Additionally, repeat measurements did not show a drift, or continuous change over time, so any reactions with the mTBD or mTBD acetate did not noticeably affect the measured values. 


\section{Results and Discussion}

\subsection{Density}

Densities were measured for 16 samples, generally in the range of $293 \mathrm{~K}$ to $363 \mathrm{~K}$. mTBD+ water samples were generally measured at only lower temperatures below $324 \mathrm{~K}$ to avoid hydrolysis, and the dry mTBD acetate samples were also measured at higher temperatures. The density data are given in Table 2, and the data have also been placed in a scientific repository (Open Science Framework) at (https://osf.io/krx5z/). The pure components had some of the lowest densities, and the density increased up to a maximum in the area where there was roughly an equal molar amount of the three compounds. Interestingly, when adding a little bit of an excess of acetic acid to the mTBD acetate the density is actually slightly higher ( $x_{\text {acid }}$ of 0.6 and no water). However, when water is present this is not observed, and the sample with excess acetic acid had a lower density than that with an equimolar ratio of acid and base. This is similar to the behavior observed with the vapor-liquid equilibrium data [12]. It has also been shown that protic ionic liquids, such as mTBD acetate, exhibit a reactive azeotrope (or possibly a complex) at a composition where there is an excess of acid, and the higher density at the composition where $\mathrm{x}_{\text {acid }}$ equals 0.6 and $\mathrm{x}_{\text {water }}$ is 0 may be due to the same intermolecular interactions that cause the azeotrope $[9,10,23]$.

The ePC-SAFT equation of state was used to model the density of this system $[24,25]$. The model was described in detail previously, which showed its performance for the vapor-liquid equilibrium (VLE) of this system [12]. 5 components were included in the model: water, mTBD, acetic acid, the mTBDH + ion, and the acetate ion. The equilibrium reaction between the acid and base was modeled using the van't Hoff equation. By including mTBD and acetic acid as components and including the reaction in the model, it is possible to also model compositions where there is an excess of the acid or base. Some of the parameters could be fit to literature data, but the remainder were optimized using the experimental density data along with the VLE data [12].

The ePC-SAFT model gives reasonable results for some compositions, but for others there are relative deviations as high as $10 \%$ (see Figs. 1 and 2). The largest errors occur for the mTBD + water system, where the densities calculated are much lower than the actual densities. This poor behavior is caused because the model had difficulty describing the simpler mTBD+ water system. When optimizing the ePC-SAFT parameters, we first fit parameters for the mTBD + water system, and even then we noticed the difficulty of getting a good fit for both the density and VLE data. To get a good fit for the VLE pressures, it appears that the equation had to be warped in a way that resulted in poor density results.

When optimization was performed using only the density data, the calculated densities were better, but the pressures were much lower than the experimental data. By comparing the parameters from the two optimizations, we could see that the kij interaction parameter between mTBD and water was less negative $\left(\mathrm{k}_{\text {base }}\right.$ of -8.028 and $\mathrm{k}_{\mathrm{T}}$ of 0.01856 ) and the lij interaction parameter was also smaller (0.198). 
Table 2 Liquid density of mixtures of mTBD, acetic acid, and water at $0.1 \mathrm{MPa}$

\begin{tabular}{|c|c|c|c|c|}
\hline $\begin{array}{l}\text { Temperature }^{\mathrm{a}} \\
\mathrm{K}\end{array}$ & $\begin{array}{l}\text { Density } \\
\mathrm{kg} \cdot \mathrm{m}^{-3}\end{array}$ & $\begin{array}{l}\mathrm{mTBD}^{\mathrm{c}} \\
\text { Mole fraction }\end{array}$ & $\begin{array}{l}\text { Water }^{\mathrm{c}} \\
\text { Mole fraction }\end{array}$ & $\begin{array}{l}\text { Acetic acid } \\
\text { Mole fraction }\end{array}$ \\
\hline 298.15 & 1087.1 & 0.3446 & 0.6554 & 0.0000 \\
\hline 293.16 & 1091.2 & 0.3446 & 0.6554 & 0.0000 \\
\hline 298.16 & 1087.0 & 0.3446 & 0.6554 & 0.0000 \\
\hline 303.15 & 1082.7 & 0.3446 & 0.6554 & 0.0000 \\
\hline 363.14 & 1101.9 & 0.4869 & 0.0000 & 0.5131 \\
\hline 356.14 & 1107.2 & 0.4869 & 0.0000 & 0.5131 \\
\hline 363.16 & 1101.9 & 0.4869 & 0.0000 & 0.5131 \\
\hline 373.16 & 1094.5 & 0.4869 & 0.0000 & 0.5131 \\
\hline 383.16 & 1087.1 & 0.4869 & 0.0000 & 0.5131 \\
\hline 393.16 & 1079.8 & 0.4869 & 0.0000 & 0.5131 \\
\hline 403.16 & 1072.4 & 0.4869 & 0.0000 & 0.5131 \\
\hline 413.15 & 1065.0 & 0.4869 & 0.0000 & 0.5131 \\
\hline 423.15 & 1057.6 & 0.4869 & 0.0000 & 0.5131 \\
\hline 363.14 & 1101.7 & 0.4869 & 0.0000 & 0.5131 \\
\hline 298.15 & 1111.7 & 0.0984 & 0.8056 & 0.0960 \\
\hline 298.15 & 1111.7 & 0.0984 & 0.8056 & 0.0960 \\
\hline 293.16 & 1114.9 & 0.0984 & 0.8056 & 0.0960 \\
\hline 298.16 & 1111.6 & 0.0984 & 0.8056 & 0.0960 \\
\hline 303.15 & 1108.2 & 0.0984 & 0.8056 & 0.0960 \\
\hline 313.16 & 1101.1 & 0.0984 & 0.8056 & 0.0960 \\
\hline 323.16 & 1093.7 & 0.0984 & 0.8056 & 0.0960 \\
\hline 333.16 & 1086.2 & 0.0984 & 0.8056 & 0.0960 \\
\hline 343.16 & 1078.7 & 0.0984 & 0.8056 & 0.0960 \\
\hline 353.16 & 1071.2 & 0.0984 & 0.8056 & 0.0960 \\
\hline 363.16 & 1063.3 & 0.0984 & 0.8056 & 0.0960 \\
\hline 298.15 & 1111.8 & 0.0984 & 0.8056 & 0.0960 \\
\hline 298.15 & 1111.7 & 0.0984 & 0.8056 & 0.0960 \\
\hline 298.15 & 1000.8 & 0.0023 & 0.9954 & 0.0023 \\
\hline 293.16 & 1001.9 & 0.0023 & 0.9954 & 0.0023 \\
\hline 298.16 & 1000.6 & 0.0023 & 0.9954 & 0.0023 \\
\hline 303.15 & 999.1 & 0.0023 & 0.9954 & 0.0023 \\
\hline 323.16 & 991.6 & 0.0023 & 0.9954 & 0.0023 \\
\hline 343.16 & 981.2 & 0.0023 & 0.9954 & 0.0023 \\
\hline 363.16 & 968.7 & 0.0023 & 0.9954 & 0.0023 \\
\hline 298.15 & 1000.8 & 0.0023 & 0.9954 & 0.0023 \\
\hline 298.15 & 1039.5 & 0.0274 & 0.9435 & 0.0291 \\
\hline 293.16 & 1041.3 & 0.0274 & 0.9435 & 0.0291 \\
\hline 298.16 & 1039.4 & 0.0274 & 0.9435 & 0.0291 \\
\hline 303.15 & 1037.2 & 0.0274 & 0.9435 & 0.0291 \\
\hline 323.16 & 1027.3 & 0.0274 & 0.9435 & 0.0291 \\
\hline 343.16 & 1015.4 & 0.0274 & 0.9435 & 0.0291 \\
\hline
\end{tabular}


Table 2 (continued)

\begin{tabular}{|c|c|c|c|c|}
\hline $\begin{array}{l}\text { Temperature }^{\mathrm{a}} \\
\mathrm{K}\end{array}$ & $\begin{array}{l}\text { Density } \\
\mathrm{kg} \cdot \mathrm{m}^{-3}\end{array}$ & $\begin{array}{l}\mathrm{mTBD}^{\mathrm{c}} \\
\text { Mole fraction }\end{array}$ & $\begin{array}{l}\text { Water }^{\mathrm{c}} \\
\text { Mole fraction }\end{array}$ & $\begin{array}{l}\text { Acetic acid } \\
\text { Mole fraction }\end{array}$ \\
\hline 363.16 & 1002.0 & 0.0274 & 0.9435 & 0.0291 \\
\hline 298.15 & 1039.5 & 0.0274 & 0.9435 & 0.0291 \\
\hline 298.15 & 1039.5 & 0.0274 & 0.9435 & 0.0291 \\
\hline 298.15 & 1153.8 & 0.2415 & 0.5057 & 0.2529 \\
\hline 293.16 & 1157.0 & 0.2415 & 0.5057 & 0.2529 \\
\hline 298.16 & 1153.4 & 0.2415 & 0.5057 & 0.2529 \\
\hline 303.15 & 1149.8 & 0.2415 & 0.5057 & 0.2529 \\
\hline 323.16 & 1135.3 & 0.2415 & 0.5057 & 0.2529 \\
\hline 343.16 & 1120.8 & 0.2415 & 0.5057 & 0.2529 \\
\hline 363.16 & 1106.3 & 0.2415 & 0.5057 & 0.2529 \\
\hline 298.15 & 1153.8 & 0.2415 & 0.5057 & 0.2529 \\
\hline 298.16 & 1153.3 & 0.2415 & 0.5057 & 0.2529 \\
\hline 298.15 & 1071.6 & 0.0529 & 0.8950 & 0.0521 \\
\hline 293.15 & 1074.1 & 0.0529 & 0.8950 & 0.0521 \\
\hline 298.16 & 1071.6 & 0.0529 & 0.8950 & 0.0521 \\
\hline 303.15 & 1068.8 & 0.0529 & 0.8950 & 0.0521 \\
\hline 323.16 & 1056.6 & 0.0529 & 0.8950 & 0.0521 \\
\hline 343.16 & 1043.2 & 0.0529 & 0.8950 & 0.0521 \\
\hline 363.16 & 1028.9 & 0.0529 & 0.8950 & 0.0521 \\
\hline 298.15 & 1071.7 & 0.0529 & 0.8950 & 0.0521 \\
\hline 298.14 & 1157.5 & 0.2914 & 0.4138 & 0.2948 \\
\hline 293.15 & 1160.8 & 0.2914 & 0.4138 & 0.2948 \\
\hline 298.15 & 1157.5 & 0.2914 & 0.4138 & 0.2948 \\
\hline 303.15 & 1153.9 & 0.2914 & 0.4138 & 0.2948 \\
\hline 323.16 & 1139.3 & 0.2914 & 0.4138 & 0.2948 \\
\hline 343.16 & 1124.8 & 0.2914 & 0.4138 & 0.2948 \\
\hline 363.16 & 1110.0 & 0.2914 & 0.4138 & 0.2948 \\
\hline 298.15 & 1157.5 & 0.2914 & 0.4138 & 0.2948 \\
\hline 323.15 & 1132.0 & 0.3909 & 0.0000 & 0.6091 \\
\hline 343.16 & 1118.2 & 0.3909 & 0.0000 & 0.6091 \\
\hline 363.16 & 1104.7 & 0.3909 & 0.0000 & 0.6091 \\
\hline 383.16 & 1091.2 & 0.3909 & 0.0000 & 0.6091 \\
\hline 403.16 & 1077.7 & 0.3909 & 0.0000 & 0.6091 \\
\hline 423.16 & 1064.1 & 0.3909 & 0.0000 & 0.6091 \\
\hline 323.15 & 1131.9 & 0.3909 & 0.0000 & 0.6091 \\
\hline 303.16 & 1146.3 & 0.3909 & 0.0000 & 0.6091 \\
\hline 298.16 & 1149.7 & 0.3909 & 0.0000 & 0.6091 \\
\hline 293.15 & 1153.0 & 0.3909 & 0.0000 & 0.6091 \\
\hline 298.15 & 1057.6 & 0.0109 & 0.7817 & 0.2074 \\
\hline 293.15 & 1061.1 & 0.0109 & 0.7817 & 0.2074 \\
\hline 298.16 & 1057.6 & 0.0109 & 0.7817 & 0.2074 \\
\hline
\end{tabular}


Table 2 (continued)

\begin{tabular}{|c|c|c|c|c|}
\hline Temperature $^{\mathrm{a}}$ & Density $^{\mathrm{b}}$ & $\mathrm{mTBD}^{\mathrm{c}}$ & Water $^{\mathrm{c}}$ & Acetic acid ${ }^{c}$ \\
\hline $\mathrm{K}$ & $\mathrm{kg} \cdot \mathrm{m}^{-3}$ & Mole fraction & Mole fraction & Mole fraction \\
\hline 303.15 & 1053.8 & 0.0109 & 0.7817 & 0.2074 \\
\hline 323.16 & 1037.8 & 0.0109 & 0.7817 & 0.2074 \\
\hline 343.16 & 1020.9 & 0.0109 & 0.7817 & 0.2074 \\
\hline 363.16 & 1003.0 & 0.0109 & 0.7817 & 0.2074 \\
\hline 298.15 & 1057.6 & 0.0109 & 0.7817 & 0.2074 \\
\hline 293.15 & 1058.0 & 0.0529 & 0.9471 & 0.0000 \\
\hline 298.16 & 1054.8 & 0.0529 & 0.9471 & 0.0000 \\
\hline 303.15 & 1051.4 & 0.0529 & 0.9471 & 0.0000 \\
\hline 308.15 & 1047.7 & 0.0529 & 0.9471 & 0.0000 \\
\hline 313.16 & 1043.0 & 0.0529 & 0.9471 & 0.0000 \\
\hline 298.15 & 1090.6 & 0.0950 & 0.8534 & 0.0516 \\
\hline 293.16 & 1094.1 & 0.0950 & 0.8534 & 0.0516 \\
\hline 298.16 & 1090.7 & 0.0950 & 0.8534 & 0.0516 \\
\hline 303.15 & 1087.1 & 0.0950 & 0.8534 & 0.0516 \\
\hline 323.16 & 1072.3 & 0.0950 & 0.8534 & 0.0516 \\
\hline 298.15 & 1067.7 & 0.8714 & 0.1286 & 0.0000 \\
\hline 293.16 & 1071.4 & 0.8714 & 0.1286 & 0.0000 \\
\hline 298.16 & 1067.6 & 0.8714 & 0.1286 & 0.0000 \\
\hline 303.15 & 1063.8 & 0.8714 & 0.1286 & 0.0000 \\
\hline 308.15 & 1059.8 & 0.8714 & 0.1286 & 0.0000 \\
\hline 313.16 & 1055.7 & 0.8714 & 0.1286 & 0.0000 \\
\hline 323.15 & 1047.4 & 0.8714 & 0.1286 & 0.0000 \\
\hline 298.15 & 1067.7 & 0.8714 & 0.1286 & 0.0000 \\
\hline 298.15 & 1085.1 & 0.1995 & 0.8005 & 0.0000 \\
\hline 293.16 & 1089.2 & 0.1995 & 0.8005 & 0.0000 \\
\hline 298.16 & 1085.1 & 0.1995 & 0.8005 & 0.0000 \\
\hline 303.15 & 1080.9 & 0.1995 & 0.8005 & 0.0000 \\
\hline 308.15 & 1076.5 & 0.1995 & 0.8005 & 0.0000 \\
\hline 313.16 & 1072.2 & 0.1995 & 0.8005 & 0.0000 \\
\hline 323.16 & 1063.5 & 0.1995 & 0.8005 & 0.0000 \\
\hline 298.15 & 1085.6 & 0.1995 & 0.8005 & 0.0000 \\
\hline 298.15 & 1081.2 & 0.5299 & 0.4701 & 0.0000 \\
\hline 293.16 & 1085.2 & 0.5299 & 0.4701 & 0.0000 \\
\hline 298.16 & 1081.2 & 0.5299 & 0.4701 & 0.0000 \\
\hline 303.15 & 1077.0 & 0.5299 & 0.4701 & 0.0000 \\
\hline 308.15 & 1072.7 & 0.5299 & 0.4701 & 0.0000 \\
\hline 313.15 & 1068.3 & 0.5299 & 0.4701 & 0.0000 \\
\hline 323.15 & 1059.5 & 0.5299 & 0.4701 & 0.0000 \\
\hline 293.16 & 1093.9 & 0.0684 & 0.8108 & 0.1208 \\
\hline 298.15 & 1090.9 & 0.0684 & 0.8108 & 0.1208 \\
\hline 298.15 & 1090.8 & 0.0684 & 0.8108 & 0.1208 \\
\hline
\end{tabular}


Table 2 (continued)

\begin{tabular}{lllll}
\hline $\begin{array}{l}\text { Temperature }^{\mathrm{a}} \\
\mathrm{K}\end{array}$ & $\begin{array}{l}\text { Density } \\
\mathrm{kg} \cdot \mathrm{m}^{-3}\end{array}$ & $\begin{array}{l}\mathrm{mTBD}^{\mathrm{c}} \\
\text { Mole fraction }\end{array}$ & $\begin{array}{l}\text { Water } \\
\text { Mole fraction }\end{array}$ & $\begin{array}{l}{\text { Acetic } \text { acid }^{\mathrm{c}}}^{\mathrm{c}} \\
\text { Mole fraction }\end{array}$ \\
\hline 298.16 & 1090.8 & 0.0684 & 0.8108 & 0.1208 \\
303.15 & 1087.5 & 0.0684 & 0.8108 & 0.1208 \\
323.16 & 1073.4 & 0.0684 & 0.8108 & 0.1208 \\
343.16 & 1058.7 & 0.0684 & 0.8108 & 0.1208 \\
363.16 & 1043.4 & 0.0684 & 0.8108 & 0.1208 \\
\hline
\end{tabular}

Standard uncertainty of pressure $\mathrm{u}($ pressure $)=0.01 \mathrm{MPa}$

${ }^{a}$ Standard uncertainty of temperature $\mathrm{u}($ temperature) $=0.1 \quad \mathrm{~K} \quad$ (expanded uncertainty $\mathrm{u}($ temperature $)=0.2 \mathrm{~K}$ at the $95 \%$ level)

${ }^{b}$ Expanded uncertainty of the density is different for each sample because purity was accounted for. The uncertainties can be found from the OSF page (https://osf.io/krx5z/). In general, the expanded uncertainty was in the range of -0.5 to $0.2 \mathrm{~kg} \cdot \mathrm{m}^{-3}$

${ }^{\mathrm{c}}$ Uncertainties of the composition of each sample can be found from the data file on the OSF page (https ://osf.io/krx5z/). The median standard uncertainty was 0.0006 and the largest was 0.0026

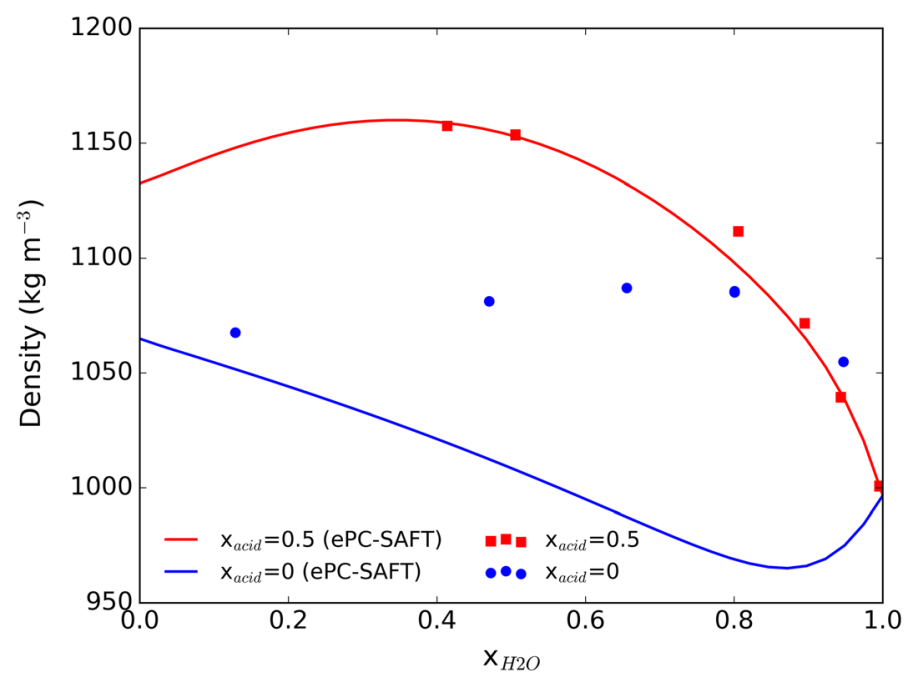

Fig. 1 Density as a function of the mole fraction of water for mixtures with an acetic acid mole fraction of 0.5 (dry basis) and mixtures with no acetic acid at $298.15 \mathrm{~K}$

Although a smaller error was obtained when including the reaction between mTBD and water in the model, including the hydroxide ion in this manner also increased the number of parameters and led to erratic results in regions between experimental data points [12]. This indicates that including these additional parameters led to a model that was too complex and likely overfit the data. 


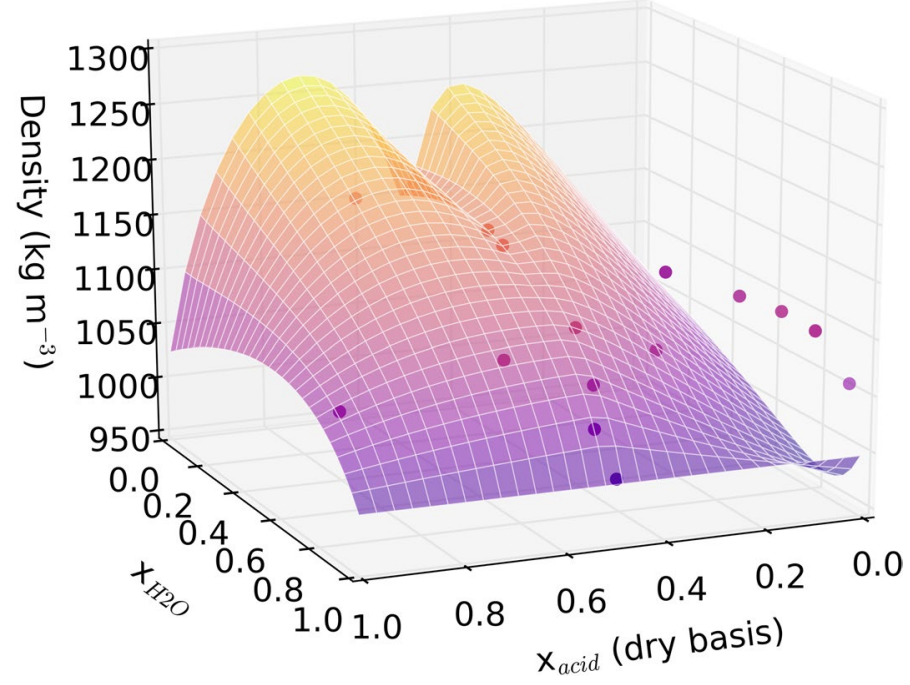

Fig. 2 Density as a function of composition for the aqueous IL mixtures at 298.15 K. The surface was calculated using the ePC-SAFT model

\subsection{Viscosity}

Viscosity was measured for 13 samples at temperatures between 294 and $365 \mathrm{~K}$. The experimental viscosity data are presented in Table 3, and can be found from the OSF project accompanying this article (https://osf.io/krx5z/). mTBD acetate (without water) was determined to have a viscosity of $12.7 \mathrm{mPa} \cdot \mathrm{s}$ at $365.0 \mathrm{~K}$. Figure 3 shows the effect of water content on the viscosity of the ionic liquid. Only samples with an equimolar ratio of mTBD and acetic acid are shown. In Fig. 3 multiple lines appear close to each other, because repeat measurements were performed with samples that had only slight differences in water content. Also, samples with little or no water were solid at room temperature, and for that reason their viscosities were only measured at higher temperatures.

We also developed a simple model to calculate the viscosity of mixtures of water and mTBD acetate. The model is only for mixtures that have an equimolar ratio of mTBD and acetic acid because most of the data we had was for this range of compositions. The model is based on Eq. 1, which is similar to the Andrade [26] and DIPPR [27] viscosity equations:

$$
\mu=\exp \left(A+\frac{B}{T}+C \cdot \ln (T)\right) .
$$

In Eq. $1, \mu$ is the viscosity $(\mathrm{mPa} \cdot \mathrm{s}), \mathrm{T}$ is the temperature $(\mathrm{K})$, and $\mathrm{A}$ through $\mathrm{C}$ are parameters for a specific mTBD acetate + water mixture. The parameter $\mathrm{C}$ was fixed at 50 for all of the samples. By fixing $\mathrm{C}$, it was possible to get the other two parameters to follow a trend with respect to the water content in the sample. Polynomials were then fit to be able to determine A and B (Eqs. 2 and 3): 
Table 3 Viscosity of liquid mixtures of mTBD, acetic acid, and water at $0.1 \mathrm{MPa}$

\begin{tabular}{|c|c|c|c|c|}
\hline Temperature $^{\mathrm{a}}$ & Viscosity $^{\mathrm{b}}$ & Acetic acid content ${ }^{c}$ & mTBD content ${ }^{\mathrm{c}}$ & Water content ${ }^{\mathrm{c}}$ \\
\hline K & mPas & & & \\
\hline 302.32 & 8.4 & 0.0752 & 0.0751 & 0.8496 \\
\hline 313.05 & 5.1 & 0.0752 & 0.0751 & 0.8496 \\
\hline 328.08 & 3.3 & 0.0752 & 0.0751 & 0.8496 \\
\hline 342.98 & 2.3 & 0.0752 & 0.0751 & 0.8496 \\
\hline 353.33 & 2.0 & 0.0752 & 0.0751 & 0.8496 \\
\hline 362.55 & 1.8 & 0.0752 & 0.0751 & 0.8496 \\
\hline 300.53 & 96.3 & 0.1960 & 0.1985 & 0.6055 \\
\hline 313.11 & 42.5 & 0.1960 & 0.1985 & 0.6055 \\
\hline 328.07 & 20.2 & 0.1960 & 0.1985 & 0.6055 \\
\hline 342.94 & 11.6 & 0.1960 & 0.1985 & 0.6055 \\
\hline 351.94 & 9.0 & 0.1960 & 0.1985 & 0.6055 \\
\hline 362.93 & 7.2 & 0.1960 & 0.1985 & 0.6055 \\
\hline 300.33 & 221.5 & 0.2963 & 0.3057 & 0.3980 \\
\hline 313.11 & 89.4 & 0.2963 & 0.3057 & 0.3980 \\
\hline 300.34 & 221.5 & 0.2963 & 0.3057 & 0.3980 \\
\hline 328.03 & 38.4 & 0.2963 & 0.3057 & 0.3980 \\
\hline 341.89 & 20.9 & 0.2963 & 0.3057 & 0.3980 \\
\hline 362.91 & 10.2 & 0.2963 & 0.3057 & 0.3980 \\
\hline 296.71 & 10.2 & 0.0704 & 0.0755 & 0.8542 \\
\hline 303.06 & 7.8 & 0.0704 & 0.0755 & 0.8542 \\
\hline 312.90 & 5.6 & 0.0704 & 0.0755 & 0.8542 \\
\hline 323.82 & 4.1 & 0.0704 & 0.0755 & 0.8542 \\
\hline 338.39 & 3.0 & 0.0704 & 0.0755 & 0.8542 \\
\hline 347.85 & 2.8 & 0.0704 & 0.0755 & 0.8542 \\
\hline 363.03 & 2.6 & 0.0704 & 0.0755 & 0.8542 \\
\hline 296.61 & 116.3 & 0.2032 & 0.1983 & 0.5985 \\
\hline 308.25 & 52.8 & 0.2032 & 0.1983 & 0.5985 \\
\hline 323.16 & 23.3 & 0.2032 & 0.1983 & 0.5985 \\
\hline 308.23 & 53.2 & 0.2032 & 0.1983 & 0.5985 \\
\hline 337.97 & 12.7 & 0.2032 & 0.1983 & 0.5985 \\
\hline 348.87 & 8.9 & 0.2032 & 0.1983 & 0.5985 \\
\hline 362.96 & 6.1 & 0.2032 & 0.1983 & 0.5985 \\
\hline 296.00 & 312.3 & 0.2994 & 0.2985 & 0.4021 \\
\hline 303.19 & 175.4 & 0.2994 & 0.2985 & 0.4021 \\
\hline 308.92 & 117.0 & 0.2994 & 0.2985 & 0.4021 \\
\hline 318.52 & 65.0 & 0.2994 & 0.2985 & 0.4021 \\
\hline 308.80 & 116.8 & 0.2994 & 0.2985 & 0.4021 \\
\hline 333.47 & 29.4 & 0.2994 & 0.2985 & 0.4021 \\
\hline 341.72 & 20.5 & 0.2994 & 0.2985 & 0.4021 \\
\hline 362.50 & 10.3 & 0.2994 & 0.2985 & 0.4021 \\
\hline 294.37 & 350.0 & 0.2955 & 0.2939 & 0.4106 \\
\hline 303.26 & 169.5 & 0.2955 & 0.2939 & 0.4106 \\
\hline 313.23 & 84.7 & 0.2955 & 0.2939 & 0.4106 \\
\hline 323.55 & 47.1 & 0.2955 & 0.2939 & 0.4106 \\
\hline
\end{tabular}


Table 3 (continued)

\begin{tabular}{|c|c|c|c|c|}
\hline $\begin{array}{l}\text { Temperature }^{\mathrm{a}} \\
\mathrm{K}\end{array}$ & $\begin{array}{l}\text { Viscosity } \\
\text { mPas }\end{array}$ & $\begin{array}{l}\text { Acetic acid content }{ }^{\mathrm{c}} \\
\text { Mole fraction }\end{array}$ & $\begin{array}{l}\text { mTBD content }^{\mathrm{c}} \\
\text { Mole fraction }\end{array}$ & $\begin{array}{l}\text { Water content }{ }^{\mathrm{c}} \\
\text { Mole fraction }\end{array}$ \\
\hline 332.71 & 29.9 & 0.2955 & 0.2939 & 0.4106 \\
\hline 348.06 & 16.1 & 0.2955 & 0.2939 & 0.4106 \\
\hline 362.87 & 10.1 & 0.2955 & 0.2939 & 0.4106 \\
\hline 343.53 & 26.5 & 0.3959 & 0.3975 & 0.2066 \\
\hline 343.54 & 26.4 & 0.3959 & 0.3975 & 0.2066 \\
\hline 359.01 & 15.0 & 0.3959 & 0.3975 & 0.2066 \\
\hline 362.65 & 13.3 & 0.3959 & 0.3975 & 0.2066 \\
\hline 362.65 & 13.4 & 0.3959 & 0.3975 & 0.2066 \\
\hline 296.80 & 23.1 & 0.0000 & 0.1982 & 0.8018 \\
\hline 303.69 & 15.8 & 0.0000 & 0.1982 & 0.8018 \\
\hline 296.87 & 24.9 & 0.0000 & 0.1982 & 0.8018 \\
\hline 313.13 & 11.5 & 0.0000 & 0.1982 & 0.8018 \\
\hline 343.25 & 6.0 & 0.0000 & 0.1982 & 0.8018 \\
\hline 362.37 & 4.4 & 0.0000 & 0.1982 & 0.8018 \\
\hline 298.43 & 11.2 & 0.0000 & 0.7980 & 0.2020 \\
\hline 308.31 & 7.6 & 0.0000 & 0.7980 & 0.2020 \\
\hline 318.19 & 5.6 & 0.0000 & 0.7980 & 0.2020 \\
\hline 343.07 & 3.0 & 0.0000 & 0.7980 & 0.2020 \\
\hline 347.15 & 2.7 & 0.0000 & 0.7980 & 0.2020 \\
\hline 362.91 & 2.0 & 0.0000 & 0.7980 & 0.2020 \\
\hline 360.84 & 14.3 & 0.5020 & 0.4980 & 0.0000 \\
\hline 364.50 & 12.8 & 0.5020 & 0.4980 & 0.0000 \\
\hline 365.00 & 12.7 & 0.5020 & 0.4980 & 0.0000 \\
\hline 294.45 & 12.9 & 0.8874 & 0.1126 & 0.0000 \\
\hline 298.17 & 11.3 & 0.8874 & 0.1126 & 0.0000 \\
\hline 308.10 & 8.1 & 0.8874 & 0.1126 & 0.0000 \\
\hline 323.06 & 5.4 & 0.8874 & 0.1126 & 0.0000 \\
\hline 343.24 & 3.6 & 0.8874 & 0.1126 & 0.0000 \\
\hline 363.04 & 2.7 & 0.8874 & 0.1126 & 0.0000 \\
\hline 294.96 & 29.2 & 0.0000 & 0.5035 & 0.4965 \\
\hline 298.43 & 23.6 & 0.0000 & 0.5035 & 0.4965 \\
\hline 308.29 & 14.0 & 0.0000 & 0.5035 & 0.4965 \\
\hline 323.16 & 7.6 & 0.0000 & 0.5035 & 0.4965 \\
\hline 342.89 & 4.2 & 0.0000 & 0.5035 & 0.4965 \\
\hline 363.12 & 2.8 & 0.0000 & 0.5035 & 0.4965 \\
\hline
\end{tabular}

Standard uncertainty of pressure $\mathrm{u}($ pressure $)=0.01 \mathrm{MPa}$

${ }^{a}$ The expanded uncertainty of the temperature increases linearly from $0.04 \mathrm{~K}$ at $293 \mathrm{~K}$ up to $0.2 \mathrm{~K}$ at $363 \mathrm{~K}$

${ }^{\mathrm{b}}$ Standard uncertainty of viscosity is $5.4 \%$ (expanded uncertainty of $12 \%$ at the $95 \%$ level)

${ }^{\mathrm{c}}$ Due to potential evaporation, it is estimated that the expanded uncertainty of the composition is likely $0.005 \mathrm{~mol}$ fraction at the highest temperature $(363 \mathrm{~K})$, and decreases proportionally to the vapor pressure at lower temperatures down to about 0.0003 at $300 \mathrm{~K}$ 


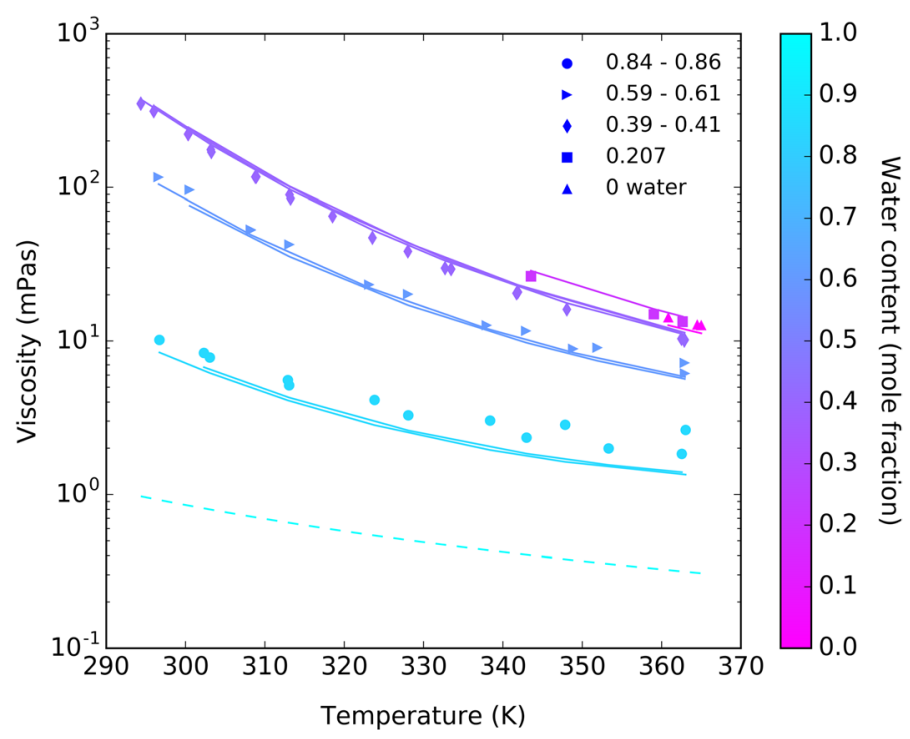

Fig. 3 Viscosity of the ionic liquid mixture as a function of water content. Only samples with equimolar ratios of $\mathrm{mTBD}$ and acetic acid are shown. Solid lines show values calculated with the model presented in this article. The dashed line shows reference values for pure water [14, 16]

$$
\begin{aligned}
& A=19.0326 \cdot x_{H 2 O}^{2}-11.3695 \cdot x_{H 2 O}-350.804, \\
& B=-8284.18 \cdot x_{H 2 O}^{2}+4511.36 \cdot x_{H 2 O}+21283.0 .
\end{aligned}
$$

In Eqs. 2 and 3, $\mathrm{x}_{\mathrm{H} 2 \mathrm{O}}$ is the mole fraction of water in the mixture. To optimize the parameters given in Eqs. 2 and 3, we first fit the parameters A and B of Eq. 1 separately for each sample from the data set. Then, using those parameter values, we regressed the constants for Eqs. 2 and 3. The root mean squared relative error of this viscosity model was $8 \%$ for the experimental IL data.

From the data and model we can see that the viscosity increases more rapidly when the IL is first added to the water, but below a water mole fraction of about 0.4 the viscosity levels out and does not vary as much with water content. This is illustrated graphically at $363 \mathrm{~K}$ in Fig. 4. Note that the points for the mixtures with no water were measured at slightly higher and lower temperatures (360 and $365 \mathrm{~K}$ instead of $363 \mathrm{~K}$ ), and so the actual value at $363 \mathrm{~K}$ would be somewhere between the points plotted in Fig. 4 .

Using the model we can also compare the viscosities of the mTBD + water mixtures to IL + water mixtures that would have the same water content. In general, the IL + water mixture has a higher viscosity. For example, at $362.9 \mathrm{~K}$ and a water content of $20.202 \mathrm{~mol} \%$ the viscosity of the mTBD + water mixture is $2 \mathrm{mPa} \cdot \mathrm{s}$ compared to $14 \mathrm{mPa} \cdot \mathrm{s}$ for an $\mathrm{IL}+$ water mixture with the same water content. 


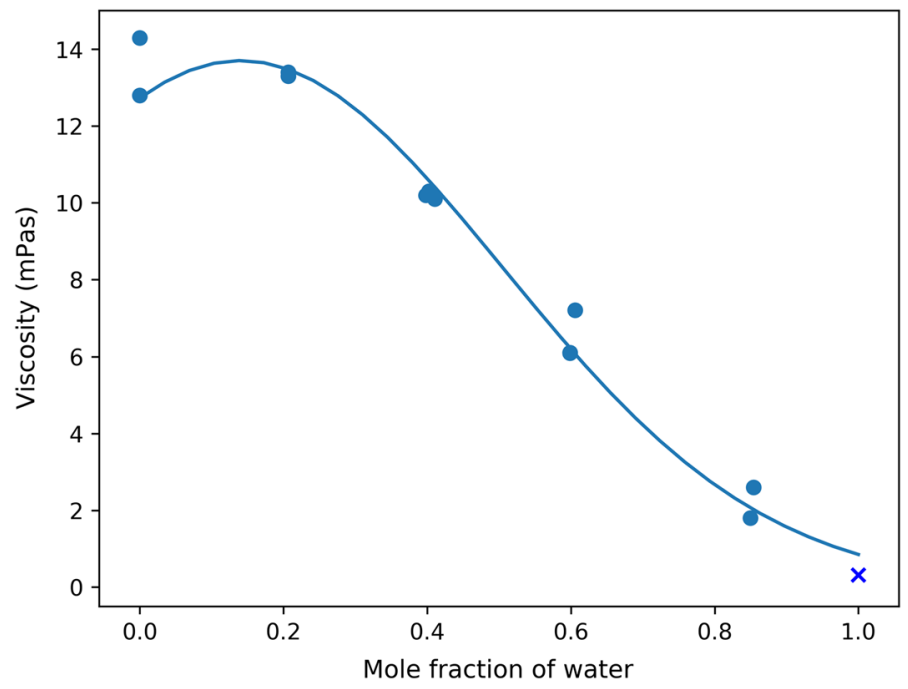

Fig. 4 Viscosity of mTBD acetate as a function of water content at $363 \mathrm{~K}$. Data points are for 360$365 \mathrm{~K}$. The $\mathrm{x}$ marker is a reference value for water $[16,28]$

\subsection{Thermal Conductivity}

The thermal conductivity was measured for three mixtures of mTBD and water and six mixtures of mTBD acetate and water. Measurements were performed between 298 and 348 or $373 \mathrm{~K}$. The experimental data are given in Tables 4 and 5, and can also be found from the OSF project accompanying this article (https://osf.io/krx5z/). The expanded uncertainty of the thermal conductivity data is $5 \%$ at the $95 \%$ confidence level. The thermal conductivity of pure mTBD was published in our earlier article [13]. Figure 5 shows the data. Water has a higher thermal conductivity, so in general, the thermal conductivity increases as the concentration of water increases. For most of the samples the thermal conductivity did not noticeably change with temperature. Also, note that the dry mTBD acetate sample is solid at the temperatures measured, which is likely the reason that two of the points for mTBD acetate are out of line with the rest of the data.

A decent estimate of the thermal conductivity can be obtained simply from a weighted average of the thermal conductivities of water and either mTBD acetate or mTBD (weight based on the mass fraction of each component). This equation, however, does overestimate the thermal conductivity by about 10 to $20 \%$. By adding an interaction parameter to the equation the root mean squared relative error was reduced to $4.7 \%$ for mTBD acetate and to $3.2 \%$ for mTBD. This equation is shown as Eq. 4:

$$
\lambda=x_{H 2 O} \lambda_{H 2 O}+x_{i} \lambda_{i}+k_{i j} x_{H 2 O} x_{i,}
$$

where $\lambda$ is the thermal conductivity of the mixture $\left(\mathrm{W} \cdot \mathrm{m}^{-1} \cdot \mathrm{K}^{-1}\right) ; \lambda_{\mathrm{H} 2 \mathrm{O}}$ and $\lambda_{\mathrm{i}}$ are, respectively, the thermal conductivities of water and the other component 


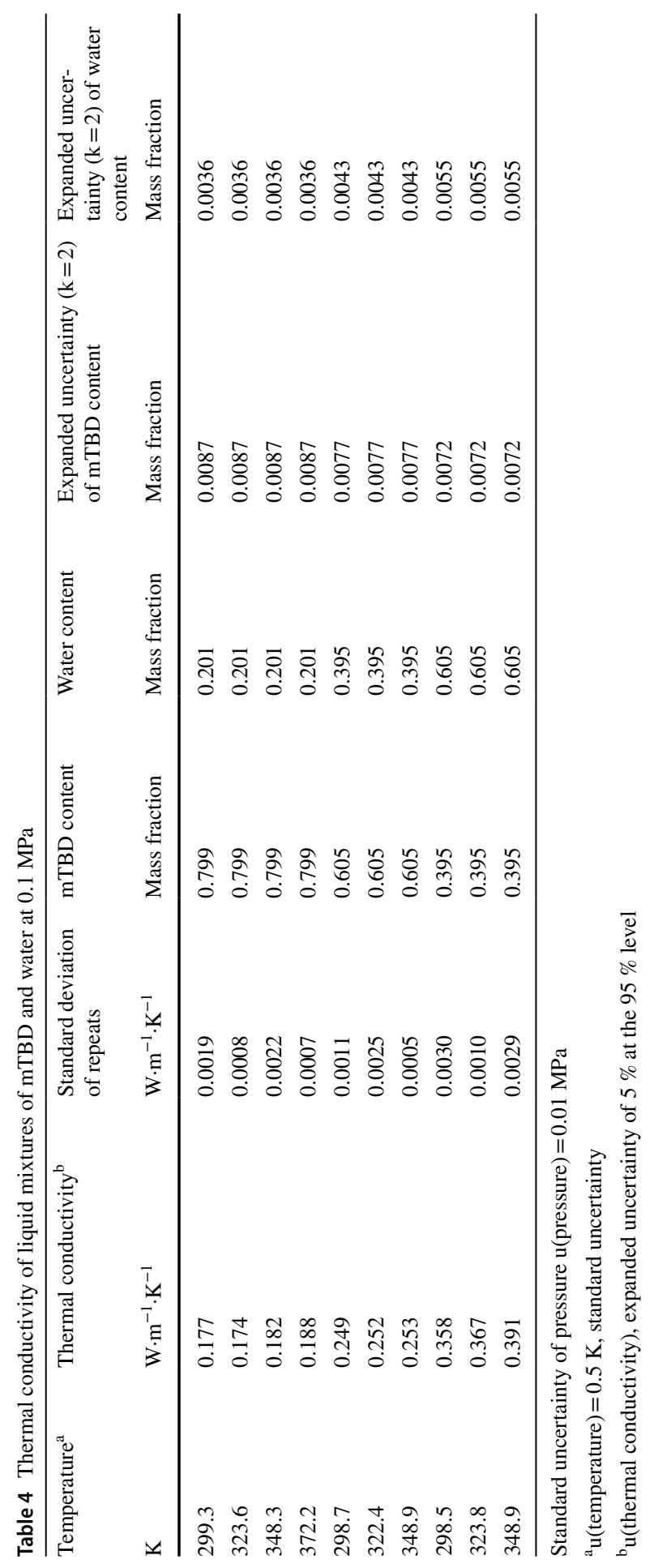




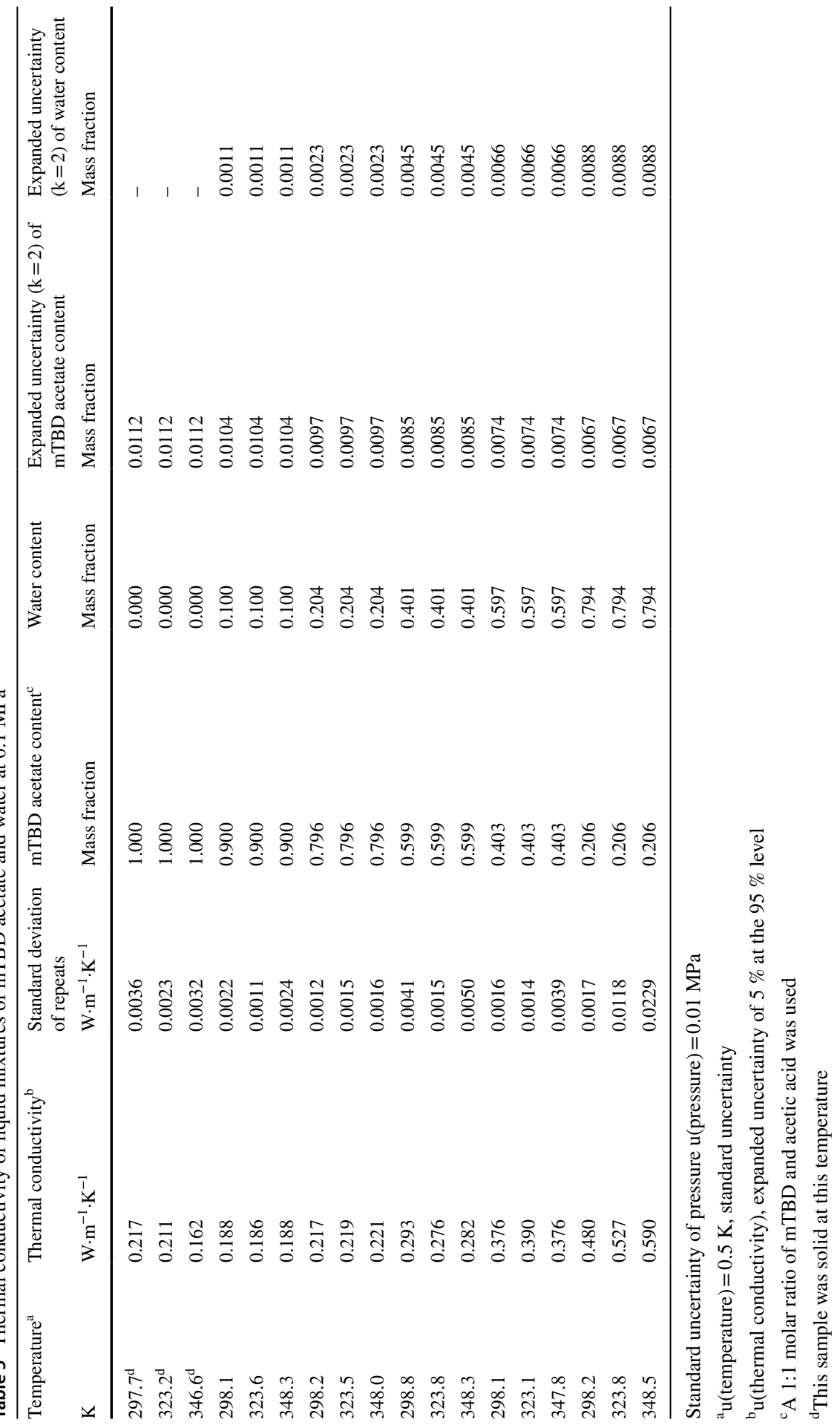




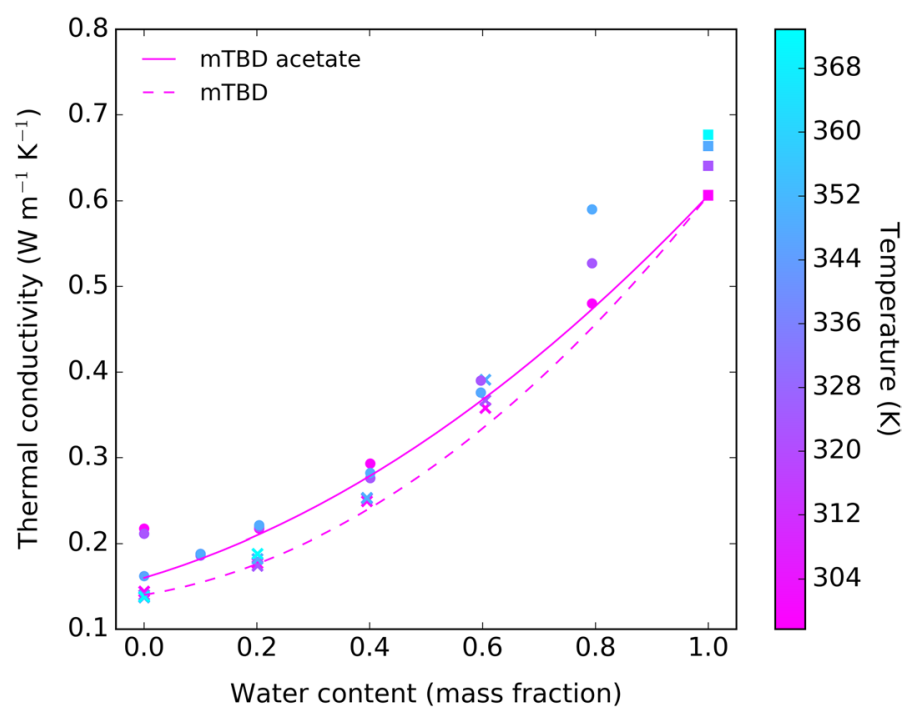

Fig. 5 Thermal conductivities of mixtures of mTBD + water (X markers) and mTBD acetate + water (dot markers). The square markers show reference values for pure water. The lines show values calculated at $298 \mathrm{~K}[16,28]$

$\left(\mathrm{W} \cdot \mathrm{m}^{-1} \cdot \mathrm{K}^{-1}\right) ; \mathrm{x}_{\mathrm{H} 2 \mathrm{O}}$ and $\mathrm{x}_{\mathrm{i}}$ are, respectively, the mass fraction of water and the other component; and $\mathrm{k}_{\mathrm{ij}}$ is an interaction parameter. For the mTBD acetate + water system $\mathrm{k}_{\mathrm{ij}}$ is -0.25 and for $\mathrm{mTBD}+$ water it is -0.357 . The thermal conductivity of pure water was calculated using the IAPWS Formulation 2011, which was implemented in the CoolProp package for Python [16, 28]. Because the thermal conductivity of mTBD acetate and mTBD seemed to vary little with temperature, we used a fixed value of $0.16 \mathrm{~W} \cdot \mathrm{m}^{-1} \cdot \mathrm{K}^{-1}$ for $\mathrm{mTBD}$ acetate and $0.14 \mathrm{~W} \cdot \mathrm{m}^{-1} \cdot \mathrm{K}^{-1}$ for $\mathrm{mTBD}$.

\section{Conclusions}

Density, viscosity, and thermal conductivity measurements were performed for the ionic liquid mTBD acetate and its mixtures with water. Samples with only mTBD and water were also measured. In general, the density and viscosity decreased as the water content increased and the thermal conductivity increased. However, at low water contents (less than a mole fraction of about 0.4 ), the density and viscosity did not vary as much with water content.

We used the ePC-SAFT equation of state to model the densities of this system. Although good results were obtained for some conditions, there were areas where ePC-SAFT gave relative errors as large as $10 \%$. Good results were obtained for mixtures with a 1:1 molar ratio of the acid and base, which is the region that is most relevant industrially. The errors were large for the system containing only mTBD and water. 
The viscosity was correlated using a simple equation with three constants. Polynomials were fit that allow these constants to be calculated based on the amount of water in the mixture, and this allows the viscosity to be calculated over a range of water contents and temperatures. The thermal conductivity could be correlated using a single interaction parameter term that is added to the weighted average of the pure component thermal conductivities.

Acknowledgment Funding was provided by Business Finland (Grant No. 560/31/2017).

Funding Open access funding provided by Aalto University.

Open Access This article is licensed under a Creative Commons Attribution 4.0 International License, which permits use, sharing, adaptation, distribution and reproduction in any medium or format, as long as you give appropriate credit to the original author(s) and the source, provide a link to the Creative Commons licence, and indicate if changes were made. The images or other third party material in this article are included in the article's Creative Commons licence, unless indicated otherwise in a credit line to the material. If material is not included in the article's Creative Commons licence and your intended use is not permitted by statutory regulation or exceeds the permitted use, you will need to obtain permission directly from the copyright holder. To view a copy of this licence, visit http://creativecommons.org/licen ses/by/4.0/.

\section{References}

1. A. Parviainen, A.W.T. King, I. Mutikainen, M. Hummel, C. Selg, L.K.J. Hauru, H. Sixta, I. Kilpeläinen, Chemsuschem 6, 2161 (2013)

2. A. Parviainen, R. Wahlström, U. Liimatainen, T. Liitiä, S. Rovio, J.K.J. Helminen, U. Hyväkkö, A.W.T. King, A. Suurnäkki, I. Kilpeläinen, RSC Adv. 5, 69728 (2015)

3. H. Sixta, M. Hummel, B. K. Le, I. Kilpeläinen, A. W. T. King, K. J. J. Helminen, and S. Hellstén, WO2018138416 (A1) (2 August 2018).

4. R.D. Rogers, K.R. Seddon, Science 302, 792 (2003)

5. Dortmund Data Bank-DDBST GmbH, https://www.ddbst.com/ddb.html. Accessed 19 Mar 2019.

6. 2018 Version of the Ionic Liquid Data Bank Available - DDBST GmbH. https://www.ddbst.com/ News-Detail/Id-2018-Version-of-the-Ionic-Liquid-Data-Bank-Available.html. Accessed 19 Mar 2019.

7. A. Kazakov, J. W. Magee, R. D. Chirico, E. Paulechka, V. Diky, C. D. Muzny, K. Kroenlein, and M. Frenkel, NIST Stand. Ref. Database 147 NIST Ion. Liq. Database - ILThermo.

8. A. George, A. Brandt, K. Tran, S.M.S.N.S. Zahari, D. Klein-Marcuschamer, N. Sun, N. Sathitsuksanoh, J. Shi, V. Stavila, R. Parthasarathi, S. Singh, B.M. Holmes, T. Welton, B.A. Simmons, J.P. Hallett, Green Chem. 17, 1728 (2015)

9. W. Ahmad, A. Ostonen, K. Jakobsson, P. Uusi-Kyyny, V. Alopaeus, U. Hyväkkö, A.W.T. King, Chem. Eng. Res. Des. 114, 287 (2016)

10. F.M.S. Ribeiro, C.F.R.A.C. Lima, A.M.S. Silva, L.M.N.B.F. Santos, ChemPhysChem 19, 2364 (2018)

11. T.L. Greaves, C.J. Drummond, Chem. Rev. 108, 206 (2008)

12. Z.S. Baird, P. Uusi-Kyyny, J. Witos, A.H. Rantamäki, H. Sixta, S.K. Wiedmer, V. Alopaeus, J. Chem. Eng. Data 65, 2405 (2020)

13. Z.S. Baird, A. Dahlberg, P. Uusi-Kyyny, N. Osmanbegovic, J. Witos, J. Helminen, D. Cederkrantz, P. Hyväri, V. Alopaeus, I. Kilpeläinen, S.K. Wiedmer, H. Sixta, Int. J. Thermophys. 40, 71 (2019)

14. W. Wagner, A. Pruß, J. Phys. Chem. Ref. Data 31, 387 (2002)

15. S. Herrmann, H.-J. Kretzschmar, D.P. Gatley, HVACR Res. 15, 961 (2009)

16. I.H. Bell, J. Wronski, S. Quoilin, V. Lemort, Ind. Eng. Chem. Res. 53, 2498 (2014)

17. R. Storn, K. Price, J. Glob. Optim. 11, 341 (1997) 
18. P. Virtanen, R. Gommers, T.E. Oliphant, M. Haberland, T. Reddy, D. Cournapeau, E. Burovski, P. Peterson, W. Weckesser, J. Bright, S.J. van der Walt, M. Brett, J. Wilson, K.J. Millman, N. Mayorov, A.R.J. Nelson, E. Jones, R. Kern, E. Larson, C.J. Carey, İ. Polat, Y. Feng, E.W. Moore, J. VanderPlas, D. Laxalde, J. Perktold, R. Cimrman, I. Henriksen, E.A. Quintero, C.R. Harris, A.M. Archibald, A.H. Ribeiro, F. Pedregosa, P. van Mulbregt, Nat. Methods 17, 261 (2020)

19. B. Kolb, L.S. Ettre, Static Headspace-Gas Chromatography: Theory and Practice (John Wiley \& Sons, Hoboken, 2006)

20. W.-Y. Wen, J.H. Hung, J. Phys. Chem. 74, 170 (1970)

21. V. Raab, J. Kipke, R.M. Gschwind, J. Sundermeyer, Chem. Eur. J. 8, 1682 (2002)

22. W. A. Wakeham, A. Nagashima, J. V. Sengers, International Union of Pure and Applied Chemistry, and Commission on Thermodynamics, Measurement of the Transport Properties of Fluids (Blackwell Scientific Publications ; Distributed in the USA and North America by CRC Press, Oxford [England]; Boston; Boca Raton, Fla., 1991).

23. J.N.C. Lopes, L.P.N. Rebelo, ChemPhysChem 12, 1948 (2010)

24. L.F. Cameretti, G. Sadowski, J.M. Mollerup, Ind. Eng. Chem. Res. 44, 3355 (2005)

25. C. Held, T. Reschke, S. Mohammad, A. Luza, G. Sadowski, Chem. Eng. Res. Des. 92, 2884 (2014)

26. E.N.D.C. Andrade, Proc. R. Soc. Lond. A 215, 36 (1952)

27. DIPPR Project 801-Full Version (Design Institute for Physical Property Research/AIChE, 2019).

28. M.L. Huber, R.A. Perkins, D.G. Friend, J.V. Sengers, M.J. Assael, I.N. Metaxa, K. Miyagawa, R. Hellmann, E. Vogel, J. Phys. Chem. Ref. Data 41, 033102 (2012) 